Nueva Revista de Filología Hispánica (NRFH), LXVIII, 2020, núm. 2, 723-746

ISSN 0185-0121; e-ISSN 2448-6558; DOI: 10.24201/nrfh.v68i2.3655

\title{
TENDENCIAS ANTIHIÁTICAS Y PROCESOS DE PALATALIZACIÓN EN EL ESPAÑOL ACTUAL: A PROPÓSITO DEL VERBO TUNEAR
}

\author{
ANTIHIATIC TENDENCIES AND PALATALIZATION \\ PROCESSES IN PRESENT-DAY SPANISH: \\ THE CASE OF THE VERB TUNEAR
}

\author{
Pilar Montero Curiel \\ Universidad de Extremadura \\ pmontero@unex.es
}

\begin{abstract}
RESUmen: Algunos procesos de evolución fonética descritos por la gramática histórica pueden rastrearse en la lengua hablada actual, sobre todo en contextos informales. Es lo que sucede con la palatalización, que ha llevado al préstamo adaptado del inglés tunear hasta la variante tuñar, no sin antes padecer la ruptura del hiato en una fase intermedia con tuniar. En esta investigación, por tanto, se pondrá de manifiesto que aún es posible profundizar en el conocimiento de estadios primitivos del idioma mediante el análisis de fenómenos paralelos, para demostrar que uno de los contextos palatalizadores de la yod segunda sigue provocando desarrollos similares a los que ocurrieron en el latín vulgar con vocablos de idéntica estructura.

Palabras clave: anglicismo; yod segunda; tendencias antihiáticas; palatalización; cambio fonético.
\end{abstract}

ABstract: Some processes of phonetic evolution described by historical grammar can still be traced in present-day language, especially in informal contexts. This is the case of palatalization which has caused the verb tunear to evolve into tunar, after passing through a previous stage as tuniar, which involves a breaking of the hiatus. This research intends to demonstrate that it is still possible to go deeper into our understanding of primitive satages of language through the analysis of parallel phenomena. In this case it serves to corroborate that one of the palatalizing contexts of second yod is still triggering processes which are similar to the ones that took place in Vulgar Latin in words with a similar structure.

Keywords: Anglicism; second yod; antihiatic tendencies; palatalization; phonetic change.

Recepción: 18 de septiembre de 2018; aceptación: 27 de marzo de 2019.

D.R. (C) 2020. Nueva Revista de Filología Hispánica Licencia Creative Commons Attribution-NonCommercial (CC BY-NC) 4.0 International 


\section{INTRODUCCIÓN}

La presencia del verbo tunear en los diccionarios de la lengua española es reciente: data de mediados del siglo xIx, y es Ramón Joaquín Domínguez quien la registra como variante de tunaren su Diccionario nacional o Gran diccionario clásico de la lengua española (1846-1847), con el sentido de 'andar vagando ocioso a la ventura en libre, suelta y holgada vida; correr la tuna con objeto vario; andar de pueblo en pueblo cantando y tocando instrumentos, pidiendo para vivir, etc.', y también 'bribonear', sujeta esta última a variación diafásica -puesto que aparece tildada de "familiar"- en sus primeras apariciones ${ }^{1}$. Hasta entonces, se encuentra documentada bajo la forma tunar desde el Diccionario de Autoridades (1739), con el sentido de 'andar vagando en vida holgazana, y libre, de lugar en lugar'. Con esta forma, llega hasta el último cuarto del siglo xIx junto a la variante modificada por el sufijo -ear, tan rentable en la formación de verbos en español a partir de diferentes bases léxicas (Martín García 2007).

En la edición de 1884 del diccionario usual, la Real Academia distingue entre la forma tunar-como 'andar vagando en vida holgazana y libre, y de lugar en lugar'- y tunear -con el sentido de 'hacer vida de tuno ó pícaro, ó proceder como tal'- con significados muy próximos, como se puede deducir de dos definiciones que evocan andanzas propias de tipos sociales, los tunos y los pícaros, muy relacionados en sus modos de vida y costumbres. Repertorios ajenos a los de la Academia, incluidos en el Nuevo tesoro lexicográfico de la lengua española, como el de Miguel de Toro y Gómez (1901), Alemany y Bolufer (1917), Rodríguez Navas (1918) o Pagés (1931), ofrecen definiciones similares de estos dos términos que llegan hasta la vigésimo primera edición del diccionario académico, la de 1992, con cambios poco llamativos. Las versiones de 2001 y 2014 ven enriquecidos sus lemas con la incorporación de nuevos usos y variantes de diversa etimología: en la primera, se incluye el verbo tunear como un mexicanismo con el sentido de 'cosechar tunas (higos de tuna)', asociado al sustantivo tuna, en referencia al fruto que da un tipo de nopal común en el país americano.

La edición de 2014 añade un tercer uso al verbo tunear como anglicismo tomado del verbo to tune, literalmente, 'afinar', 'ajustar', término asociado al lenguaje musical, que ha pasado al español como un verbo transitivo con el sentido de 'adaptar algo, especialmente

1 Las referencias a los diccionarios antiguos del español, desde el de Autoridades hasta la edición de 1992 del diccionario usual, provienen del Nuevo tesoro lexicográfico de la lengua española, consultado en la página de la Real Academia Española (http:// ntlle.rae.es) el 11 de marzo de 2018. 
un vehículo, a los gustos o intereses personales'². Esta última, por su aspecto patrimonial y su homonimia con un verbo existente en el repertorio de la lengua española, se ha incorporado al léxico de nuestro idioma sin indicios de exotismo en virtud del sufijo verbal -ear, con el que ha conseguido fijar su forma, como lo han hecho otros muchos verbos a lo largo de la historia de la lengua española "a partir de sustantivos y adjetivos, pero también de algunos pronombres y de otras clases de palabras" (NGLE 2009, p. 587). El que nos ocupa, tunear, forma parte de ese conjunto de verbos terminados en -(n)ear que derivan de préstamos tomados de otros idiomas, entre los que predomina el inglés. La mayoría de estos verbos, según la gramática académica, se origina a partir de sustantivos (es decir, tienen bases nominales) y se agrupa en torno a tres parcelas de significado: el deporte (batear, golear, boxear), la informática (chatear, formatear, resetear) y los medios de comunicación y reproducción gráfica (escanear, faxear, zapear) (NGLE 2009, p. 600; Pena 1980 y 1993; Serrano Dolader 1999; Martín García 2007).

Como apunta el diccionario académico, tunear hace referencia a la acción de transformar un objeto, sobre todo un vehículo, para adaptarlo a los gustos personales de su propietario, a partir del verbo to tune que significaba en su origen la acción de modificar parte de un instrumento musical para mejorar su sonido y, más tarde, hacer cambios en los componentes de un motor para perfeccionar sus funciones $^{3}$. Con él se relacionan los derivados tuneo y tuneado, procedentes de las mismas bases inglesas, en este caso del gerundio tuning (de to tune up), que, en referencia a un motor, significa 'ponerlo a punto'. Ambos vocablos, acomodados ya bajo las formas tuneo y tunear, han pasado a los diccionarios actuales de la lengua española "sobre el modelo de otros verbos y sustantivos de nuestra lengua que proceden del inglés, del tipo to reset, 'resetear' y 'reseteo' o to scan, 'escanear' y "escaneo"" , y se han acoplado en todas las regiones del ámbito hispano con variantes geográficas si se los juzga desde una perspectiva fonética. El Diccionario de americanismos de la RAE (2010, s.v. tunear) identifica en Ecuador la acepción juvenil y espontánea de 'embellecerse una persona' gracias a la cirugía o al maquillaje.

En los usos orales de buena parte del dominio lingüístico del español, el verbo tunear está sujeto a procesos de variación en los que desempeña un papel primordial la articulación del hiato formado por la unión de las vocales $e$ y $a$, la primera de abertura media y localización palatal, y la segunda, de abertura máxima y localización central.

\footnotetext{
${ }^{2}$ Con el mismo sentido lo registran Manuel Seco et al. (2018 [1999]), s.v. tunear.

${ }^{3}$ Véase https://dictionary.cambridge.org/es/diccionario/ingles/tune (consultado el 8 de mayo de 2018).

${ }^{4}$ Fundéu, https://www.fundeu.es (consultado el 1 de febrero de 2018).
} 
El español medio-culto, fiel a los dictados de la norma, aconsejaría la pronunciación de los dos elementos del hiato; pero en el español hablado es muy marcada la inclinación hacia el cierre del primer elemento vocálico para convertir la secuencia en un diptongo que origina la variante tuniar, analógica con un repertorio limitado de verbos que contienen la secuencia-niar etimológicamente (acalumniar, caloniar, calumniar, congeniar, endemoniar, desendemoniar, herniarse, ingeniar, matrimoniar, miniar y testimoniar, según los registros del DLE) y pueden servir de modelo a articulaciones similares originadas por la fuerte tendencia antihiática del español hablado en algunas zonas de su amplio dominio geográfico.

Con los planteamientos anteriores, el objetivo del presente estudio es describir el cambio fonológico que ha llevado al anglicismo tunear a experimentar un proceso de palatalización, tras la ruptura de su hiato, semejante al que ocurrió en el latín vulgar con vocablos de estructura y características análogas. En este proceso se pueden distinguir dos etapas: en primer lugar, la ruptura del hiato tras el cierre en un grado de la vocal palatal /e/, que modifica su articulación y pasa a adquirir rasgos semiconsonánticos y, en segundo, la palatalización del fonema /n/ atraído por esa yod semiconsonántica originada por la diptongación del hiato. De esta evolución resultan en el español hablado varias soluciones: tuniar [tu'njar], tuñear [tu'near], tuñiar [tu'njar] y tuñar [tu'nar]. La primera se justifica por la tendencia antihiática del español; la segunda y la tercera son soluciones híbridas, fruto de dos procesos: por un lado, la conservación del hiato y luego su diptongación; por otro, la palatalización del fonema alveolar sonoro nasal /n/, que se aprecia en la escritura de las dos formas; la tercera culmina el proceso de palatalización de la consonante y la fusión plena de la semiconsonante con esa nueva palatal. Entre las cuatro, predomina la primera en una proporción llamativa dentro de la documentación allegada.

Las formas anteriores (y la base de todas ellas, tunear) han permitido confeccionar un inventario que ha partido de una primera consulta en el CORPES XXI, donde aparecen 43 registros de tunear en 38 documentos procedentes de Argentina, Ecuador, España, México, Paraguay, Puerto Rico y Uruguay, datados entre 2002 y 2016. Este inventario, que ofrece 31 formas del verbo conjugado (todas ellas con hiato según corresponde a la norma escrita de la lengua, como tunee, tuneemos, tuneada, tunea, has tuneado, habia tuneado) y 12 del infinitivo (tunear), incorpora una emisión radiofónica grabada en Puerto Rico el 24 de abril de 2002 en la que se percibe con nitidez la realización del diptongo /ja/, derivado de la tendencia antihiática que caracteriza en el registro oral del español los usos del verbo que nos ocupa: “¡Ay, que tengo que tuniar [tu’njar]!”, exclama el doctor Selástraga, personaje de ficción interpretado por Antonio Sánchez, alias "El 
Gángster", en un momento del audio ${ }^{5}$. El testimonio resulta interesante porque ayuda a confrontar la norma escrita, conservadora del hiato -en la transcripción del texto se ha optado por tunear-, con la norma oral, que cierra la vocal /e/ en un grado hasta convertirla en una semiconsonante palatal, prenuclear ahora con respecto a la vocal plena o núcleo silábico.

Las 43 formas del corpus académico dan fe, además, de la rápida expansión del anglicismo tunear en el ámbito hispanohablante. Todas proceden de textos escritos de diversa índole (periodísticos, literarios, folletos, transcripciones de programas de radio o televisión) y ponen de manifiesto que en la actualidad el vocablo ha trasladado sus esferas de uso y ha pasado de denotar la acción de 'modificar vehículos' (autos, aviones, motocicletas) a representar, en el ámbito coloquial, cualquier transformación ejercida sobre un objeto para cambiar su aspecto o sus rasgos originales, como demuestran los siguientes ejemplos ${ }^{6}$ :

1. Quiero sentirme libre de tunear, programar, configurar, instalar y borrar (Argentina, 2010).

2. Laurie, tuneado como un caballero inglés, hace mímicas en el micrófono (Argentina, 2012).

3. Todo lo que hice [en la posproducción de una película] ellos lo tunearon (Ecuador, 2007).

4. Maggy, la mujer más tuneada de la empresa (España, 2007).

5. Si queremos que nos tunee un perfume creado a nuestra imagen y semejanza hemos de pedirle hora (España, 2008).

6. Podemos tunear la receta añadiendo piñones (España, 2009).

7. Desmontar a Marsé -un autor prolijo en imágenes, amante de las mismas- y volver a montarlo. Y luego contárselo al autor. Coloquialmente, ¿tuneamos a Marsé? Es el momento, porque ¿cómo se tunea un libro electrónico? (España, 2009).

8. Así concluyó, con emotivas palabras, la activa, exuberante y recién tuneada candidata (Paraguay, 2012).

Otras formas mantienen el significado original del anglicismo, fiel a sus primeras adaptaciones:

${ }^{5}$ Véase http://web.frl.es/CORPES/org/publico/pages/consulta/entradaCompleja.view (consultado el 7 de marzo de 2019).

${ }^{6}$ Véase la procedencia de los ejemplos en el Apéndice. 
1. Los personajes y la cuestión dramática de fondo no tienen mayor sustento, por lo que todo funciona como un buen pie para que Vin Diesel golpee gente de un lado para otro, se meta en medio de fiestas con chicas bailando en la calle contra autos tuneados y corra unas cuantas carreras de vértigo (Uruguay, 2009).

2. Como advertencia: si has tuneado tu coche, le has colocado accesorios que no son de serie o le has hecho cualquier "mejora", es muy posible que no te lo cubran o que te cueste un ojo de la cara (México, 2014).

3. Con este primer curro pudo comprarse un coche al que no tardó en cambiarlo completamente por dentro y decorarlo a su gusto. Lo que ahora llamamos despectivamente tuneo (España, 2015).

Al tratarse de formas escritas, dan pistas sobre la extraordinaria expansión del término en español y su popularidad en las dos orillas del Atlántico, pero no reflejan procesos de cambio fonológico, salvo el de Puerto Rico, por mostrar solamente representaciones gráficas afines a los modelos normativos.

Es justo manifestar en estas páginas introductorias que la variante que dio pie a la búsqueda de formas relacionadas con el anglicismo tunear -y a pensar que en el español actual es posible encontrar fenómenos de evolución paralelos a los que determinaron ciertos procesos de fonologización en el latín vulgar- fue la frase exclamativa "¡lo tuñé!", hallada en el Facebook de una artista argentina que divulga collages en los que suele transformar objetos cotidianos ${ }^{7}$. Este hallazgo estimuló el rastreo de las posibles variaciones articulatorias del verbo tunear (tuniar, tuñear, tuñiar, tuñar) en diversas fuentes orales y escritas. El buscador Google permitió reunir un inventario copioso de variantes útiles para reconstruir el paradigma en los distintos tiempos, modos y personas en español, si bien la palabra clave de cada búsqueda fue el infinitivo. La mayoría de ellas se encuentra en vídeos y en foros de aficionados a la transformación (o "costumización”, como también suelen llamarla) de vehículos, sobre todo de motocicletas y automóviles. Otras fueron obtenidas desde las redes sociales (Facebook, Instagram) o desde YouTube, que ha proporcionado algunos de los testimonios orales más interesantes para llevar a cabo el presente estudio. La facilidad con la que Internet permite establecer conexiones y la infinidad de caminos diferentes que abre la búsqueda de cualquier vocablo han ayudado a incrementar el exiguo corpus inicial. Las pesquisas descritas han permitido trabajar con un total de 55 variantes del verbo tunear que pueden clasificarse así:

${ }^{7}$ Cf. https://www.facebook.com/teresa.sarandon (consultado el 10 de enero y el 24 de mayo de 2018). 
a) 17 ejemplos relacionados con tuniar que reflejan el primer estadio, la conversión del hiato en diptongo.

b) 15 de tuñiar, con ruptura del hiato y un proceso fonológico de palatalización (la articulación se retrasa desde la zona alveolar al paladar duro) del fonema alveolar sonoro nasal.

c) 14 de tuñear, forma conservadora del hiato, con palatalización del fonema alveolar sonoro nasal.

d) 9 de tuñar con palatalización del fonema /n/ del verbo tunear y fusión del segmento nasal y la semiconsonante palatal tras la fractura del hiato ${ }^{8}$.

Esta variedad invita a reflexionar sobre la rápida adopción de un préstamo del inglés al español actual y sobre la posibilidad de que, en muchos casos, su entrada en la lengua se haya producido por vía oral y se haya visto influida por la tendencia antihiática de nuestra lengua. Ambas circunstancias favorecerían la palatalización tras la ruptura del hiato que se sitúa en la primera fase del fenómeno y que puede evocar, en un contexto diferente y muy alejado en el tiempo, algunos procesos de fonologización ocurridos en el paso del latín a los romances.

\section{LAS TENDENCIAS ANTIHIÁTICAS \\ EN EL ESPAÑOL ACTUAL: DE TUNEAR A TUNIAR}

Tunear en español es un verbo formado por la unión de tres sílabas (tu-ne-ar): las dos primeras, libres, y la tercera, trabada por la consonante $-r$, que está en posición marginal posnuclear. Las tres sílabas de la secuencia tienen sus núcleos vocálicos (pues son vocales plenas, según la terminología de la RAE 2011, p. 333) y sus márgenes consonánticos, estos últimos en distintas situaciones con respecto a los núcleos. Se trata de una estructura en la que se descubren dos vocales contiguas que forman parte de sílabas diferentes y originan un hiato por la confluencia de una vocal palatal de abertura media (la $e$ ) y otra de localización central y abertura máxima (la $a$ ). Ambos elementos mantienen, sin alterarlos, sus rasgos articulatorios en buena parte del dominio hispánico entre hablantes cultos, pues, como es bien sabido, en este grupo "la tendencia predominante es la aproximación a los estándares literarios escritos" (Lipski 1996, p. 166). Pero en la lengua hablada se observa una inclinación a la ruptura del hiato (Aguilar

${ }^{8}$ El intento de localizar estas palabras en los diferentes países del entorno hispanohablante no fue sencillo ni directo. La ubicación de los casos obtenidos se ha determinado después, una vez leído y organizado el material, configurado el corpus y analizados sus componentes. Tras la identificación de muchas de las formas objeto de estudio hay abundantes horas de audición y transcripción de documentos sonoros (sobre todo de vídeos de YouTube). 
1999 y 2006, p. 355), es decir, a la unión en una sola sílaba de elementos vocálicos que pertenecen a dos sílabas diferentes o, por decirlo con las palabras de Antonio Quilis (1999, pp. 189-190, y 2005, p. 107), a la conversión de los hiatos (secuencias heterosilábicas) en diptongos (secuencias o combinaciones tautosilábicas). De esta tendencia resulta en español la variante tuniar [tu'njar] con refuerzo del límite silábico formado por la unión de las dos vocales y la transformación del núcleo vocálico de la segunda sílaba en una semiconsonante palatal $/ \mathrm{j} /$ que asimila los rasgos propios del margen silábico, al no ser vocal plena; desde esta posición, el vocablo convierte su hiato en un diptongo y ve modificada su estructura: pasa de ser trisílaba a bisílaba, aunque, como es lógico, mantiene la acentuación oxítona, puesto que la intensidad sigue recayendo sobre el núcleo vocálico.

El proceso descrito se encuentra en las propias bases etimológicas del castellano, como tendencia natural del latín hablado que originó algunos de los cambios más importantes en el paso a las lenguas románicas: la predisposición a agrupar en una misma sílaba las vocales en contacto. En este proceso se sitúan algunas de las bases latinas que favorecieron palatalizaciones consonánticas por la acción de determinados tipos de yod descritos por Menéndez Pidal (1904) y por otros historiadores de la lengua española: PUTEU $>$ ['putju ] > pozo; ARANEA > [a'ranja] > araña; PALEA > ['palja] > paja, por citar algunos ejemplos representativos.

Si se toma como base esta perspectiva diacrónica, cabe afirmar que en el anglicismo tunear, asimilado por el español actual, la ruptura del hiato provoca la unión de un fonema alveolar sonoro nasal /n/ con la yod y acarrea una variante (en el ámbito de lo oral, primero) de estructura fónica análoga a la documentada en voces cultas latinas del tipo compania, hispania, ingeniu, testimoniu, iuniu, somniu y también en las pronunciaciones latinovulgares de formas como arania (a partir del latín clásico aranea), cunia (desde cunea), castania (desde castanea) y otras similares. Algunas de ellas se encuentran en la lista de incorrecciones del latín condenadas por el Appendix Probi, con diptongos originados también por tendencias antihiáticas normales en esta variante diafásica de la lengua culta de Roma: "vinea non vinia", "tinea non tinia", "cavea non cavia" y en ultracorrecciones del tipo "lilium non lileum", "noxius non noxeum", "tolonium non toloneum", que hablan de la notable vitalidad de un cambio en el uso hablado al que la norma escrita no consiguió hacer frente. Este paso, como indica Väänänen (1971, p. 86), comenzó a manifestarse en fechas

${ }^{9}$ El mismo fenómeno se descubre en secuencias diferentes, como evidencian ejemplos del tipo "brattea non brattia", "balteus non baltiius", "cochlea non coclia", "cavea non cavia", "lancea non lancia", "calceus non calcium", todas ellas en el Appendix Probi (VÄÄNÄNEN 1971, p. 86). 
muy tempranas y "está asegurado por el romance común", con testimonios en la mayoría de las lenguas derivadas del latín.

Si volvemos la vista hacia los procesos fonológicos que desencadenaron la crisis del sistema consonántico latino, observaremos cómo estas pronunciaciones peculiares de determinados hiatos favorecieron contextos fónicos que acabarían originando palatalizaciones bien estudiadas por los historiadores del latín vulgar y de las lenguas romances, como se indicó más arriba. Menéndez Pidal explica que la conservación de los hiatos latinos fue excepcional y señala que en el paso al romance predomina la tendencia a romper el hiato con diferentes procedimientos (1985 [1904], pp. 82-83). En la misma línea, Manuel Ariza recuerda que en el latín hablado era muy activa la propensión a convertir los hiatos en diptongos. En estas tendencias se sitúa el origen de algunas de las transformaciones que provocaron el surgimiento de un haz consonántico asociado a la zona articulatoria del paladar duro, que ningún otro sonido había logrado ocupar (Ariza 2012, p. 26). Antes de alcanzar esa región, el latín vulgar logró modificar el límite silábico de determinadas secuencias vocálicas y cerró las vocales de abertura media, tanto en el orden palatal $(\mathrm{e}>\mathrm{i})$ como en el velar $(\mathrm{o}>\mathrm{u})$, para mutar así los hiatos originarios en diptongos (Quilis 2005, p. 70) ${ }^{10}$.

Los procesos mencionados permiten entender el paso del latín clásico calceus al vulgar calcius, de cochlea a coclia, o de solea a solia, por citar tres entre los abundantes ejemplos ilustrativos que ofrecen contextos de palatalización. El cambio que representan es, como he expresado en otro lugar (Montero, en prensa), idéntico al que se descubre hoy en transformaciones del tipo Joaquín > *Juaquin [xwa'kin]; cohete $>$ *cuete ['kwete]; línea $>$ *linia ['linja]; alineación $>$ *alineación

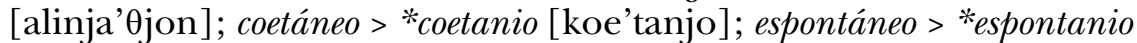
[espon'tanjo]; delineante $>$ *deliniante [deli'njante]; ser la hostia $>$ ser *laustia ['laustja]; el apelativo *hijueputa [ihwe'puta] a partir de 'hijo de puta', formas que conviven en la actualidad con ultracorrecciones del tipo arreate por arriate o geráneo por geranio.

En este mismo sentido, el verbo tunear articulado como tuniar [tu'njar] muestra una modificación en su estructura silábica (de tu-near [tu'near] a tuniar [tu'njar]) por el rompimiento del hiato originario, de forma que la vocal situada en el margen silábico (prenuclear) comienza a comportarse como una yod semiconsonántica (Quilis

${ }^{10}$ Ya se ha dicho que estos cierres, atestiguados en fechas muy tempranas en escritores cultos, se reflejan también en inscripciones primitivas, algunas del siglo II a.C., como la Ley de Bantia, de la época de los Gracos, donde se encuentra el cambio Tiano por Teano, o algunos textos de Pompeya, también citados por Väänänen, que proporcionan ejemplos de esta mutación primitiva: aenia por a(h)enea, o casium, putiolus, habias, entre otros (VÄÄNÄNEN 1971, pp. 85-86). 
2005, p. 70) en el sociolecto o variedad diastrática de determinados grupos de hablantes:

1. Cómo tuniar una zanella. Hola gente hoy les voy a enseñar a tuniar una zanella (Argentina).

2. Vendo fiat para coleccionista o para aquellos que le gusta tuniar veículos (La Rioja, Argentina).

3. Vendo todo esto para tuniar moto (Córdoba, Argentina).

4. Cómo tuniar tu auto de gta san andreas (Chile).

5. Kit completo de cromado para tuniar vehículos (Colombia).

6. Se vende auto ideal para tuniar (Uruguay).

7. Barras para tuniar tu auto (Uruguay).

8. Aprende a tuniar tu moto en 2 minutos (México). [Este vídeo provoca una cincuentena larga de comentarios entre los que aparece otras tres veces el verbo tuniar].

9. Cómo tuniar cpu (México).

10. Cómo encontrar la Miniguin y cómo tuniar autos no MD PS2 (México).

11. Cómo tuniar coche saltarín en gta san andreas: aquí les dejo la clave de arreglar coches por si asen tira el auto cuando está tuniado (México).

12. Ayuda a comensar a tuniar un fiat punto (México).

13. Dónde tuniaruna greddy ultimate y su precio (hispano residente en Estados Unidos).

14. GTA5 Cómo Tuniar El Auto De Franklin (hispano residente en Estados Unidos).

15. En éstas el patrón ha parado el motor y se acerca a la proa, mirando preocupado el costado herrumbroso de un viejo barco de hierro que está amarrado junto a él. Tampoco hace el menor esfuerzo introductorio en lengua aborigen. Itis tuniar, dice a palo seco. ${ }_{i}$ Haventyu a beterpleis? Y en ese momento pienso yo: tiene huevos aquí, el almirante (España).

16. Tuniar auto (sin ubicación). 
17. help gta san andreas (cómo tuniar autos) tuto para aprender tuniar autos cool. Autos mejor tuniados (sin ubicación).

Los ejemplos anteriores muestran que la variante tuniar [tu'njar] está presente en distintas áreas y regiones del idioma, con predominio en Argentina (Colantoni \& Limanni 2008) y México:

TABLA 1

\section{La variante tuniar}

\begin{tabular}{lc}
\hline Argentina & $3(17.64 \%)$ \\
Chile & $1(5.88 \%)$ \\
Colombia & $1(5.88 \%)$ \\
México & $5(29.41 \%)$ \\
España & $1(5.88 \%)$ \\
Estados Unidos & $2(11.76 \%)$ \\
Uruguay & $2(11.76 \%)$ \\
Sin ubicación & $2(11.76 \%)$ \\
Total & 17 \\
\hline
\end{tabular}

Desde un punto de vista fonético, confirman impresiones apuntadas por los estudiosos del español a ambos lados del Atlántico acerca de la "marcada tendencia antihiática que conduce a la pronunciación como diptongos de los hiatos", según se lee en la Fonética y fonología de la Real Academia Española (2011, p. 339), que interpreta pronunciaciones del tipo teatro $>*$ tiatro, cohete $>* c u(h)$ ete como variantes "en función del estilo o del registro", muchas veces marcadas por la influencia de factores como "el dialecto, el grado de formalidad o la velocidad de elocución". La propia Academia, en su Diccionario panhispánico de dudas, insiste en que tales articulaciones son frecuentes "en hablantes poco instruidos", al tiempo que aconseja evitarlas, pese a que "en algunos países de América, particularmente en México, este cierre vocálico se da también en el nivel culto" (2005, s.v. hiato).

Frente a esta variación, en el resto de la comunidad hispana la norma de prestigio adoptaría tunear sin restricciones, como evidencian los más de 40 casos hallados en la base de datos académica CORPES XXI en textos de diversa tipología, según se indicó en las páginas introductorias. Todos ellos dan cuenta de la importante presencia que ha alcanzado el vocablo en textos españoles e hispanoamericanos actuales entre los años 2002 y 2016, mucho antes de que el repertorio académico le abriera sus puertas:

1. En la sociedad de la información y con la tecnología disponible, pretender que no copiemos, reciclemos y tuneemos es como poner puertas al campo (Ecuador, 2006). 
2. Cada ecosistema, perfectamente tuneado a sus condiciones particulares, interactúa a su vez con otros ecosistemas (España, 2006).

3. Fiestas con chicas bailando en la calle contra autos tuneados (Uruguay, 2009).

Pese a la uniformidad que ofrecen los ejemplos proporcionados por esta base de datos, el vocablo elegido para ilustrar las tendencias actuales hacia la ruptura del hiato no representa un caso aislado circunscrito a determinadas capas de hablantes. Los estudios sobre la fonética del español actual localizan el fenómeno en diferentes áreas geográficas, como puede verse en los diferentes capítulos del Manual de dialectología hispánica, coordinado por Manuel Alvar en 1996, que proporciona casos abundantes de esta tendencia articulatoria en España y en América, desde México hasta la Tierra del Fuego, y en otros estudios centrados en la fonética hispánica, como el de María Vaquero (1996, p. 13) ${ }^{11}$.

Cabe afirmar, por lo tanto, que la ruptura del hiato constituye un proceso habitual en el español a ambos lados del Atlántico. Antonio Alcoholado (2013, p. 9) interpreta con criterios fonéticos la valoración normativa que se ha hecho tradicionalmente de esta tendencia al partir de la convicción de que, cuando se fractura el hiato, se produce una diptongación en la que una de las vocales plenas funciona como núcleo silábico, y la otra, como margen. En su opinión, tal circunstancia "contradice el precepto gramatical de que solamente las vocales cerradas $i$ y $u$ pueden situarse en margen de sílaba" $(i d$.). Esta circunstancia también se deduce de las tesis de Tomás Navarro Tomás (1918) y Antonio Quilis (1999), para quienes "se trata de un hecho constante del habla", o de la Fonética de la RAE (2011, y antes, Emilio Alarcos 1971), que entiende el fenómeno como un uso vulgar del

11 María VAQUero (1996a, p. 55), por ejemplo, encuentra en el habla antillana claras muestras del proceso en vocablos como * pasiar por paseary * ariopuerto por aeropuerto, con metátesis de yod. También en México describe Lope Blanch (1996, p. 82) una propensión similar en el habla, que considera relativamente frecuente incluso en el nivel culto (pese a la opinión de la RAE 2005, s.v. hiato): "la diptongación de los hiatos /ea/, /eo/, /oa/, /oe/" en formas como *tiatro por teatro, *pior por peor, *tuaya por toalla, *pueta por poeta, "que otras normas hispánicas rechazan como vulgares". En América Central, Quesada Pacheco (1996, p. 103) observa que es muy habitual la eliminación de los hiatos para convertirlos en diptongos, en grupos cuyo acento recae sobre la segunda vocal: patear $>*$ patiar, peor $>*$ pior, cohete $>*$ cuete. En el habla espontánea de Venezuela, SEdano y Bentivoglio (1996, p. 120) descubren sinéresis parecidas de las secuencias vocálicas /ea/ (real >*rial), /ee/ (planteé > *plantié), /eo/ (peón >*pión) y /oa/ (toalla >*tualla). El fenómeno no es ajeno a la costa caribeña de Colombia (Montes Giraldo 1996, p. 136) y aparece de manera esporádica en Paraguay, en el adjetivo * pior en vez de peor, registrado por Manuel Alvar (1996, p. 200), que sitúa este ejemplo al lado de otros como *almuada por almohada, *cuete

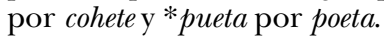


español de España y lo admite como "rasgo culto" del de América. Esta posición, como muy bien explica Alcoholado (2013, p. 9), provoca controversias razonables al interpretar como rasgo culto del español americano lo que en el europeo se considera vulgar.

Con datos procedentes del disco publicado por la Real Academia Española como anexo de su Fonética y fonología del español (2011), Las voces del español. Tiempo y espacio, Alcoholado (2012 y 2013) observa que en el mundo hispanohablante se adivinan variados "fenómenos de resilabación y de tendencia antihiática" que configuran "rasgos estándares del habla”. Desde esta perspectiva, encuentra que la tendencia antihiática del español encaja en la posible dicotomía lengua hablada/ lengua escrita, y pone de manifiesto que la predisposición apuntada no es ajena al habla de las diferentes regiones del idioma y que es normal encontrar sus reflejos en todo tipo de hablantes. Queda claro, por tanto, que la lengua hablada es capaz de transgredir los dictados de las reglas gramaticales para favorecer que algunas vocales en situación de hiato puedan reducirse a una sílaba o, mejor, a una secuencia monosilábica. La Real Academia Española (2011) insiste en que esta tendencia a quebrar el hiato se da "especialmente en el habla rápida” (p. 349) y en ciertas variedades del español con diversos grados de aceptación social (p. 353). Así lo corroboran los ejemplos de la variante tuniar, representados (sin excepción) en grabaciones y transcripciones muy breves que sirven para dar título a los vídeos (por ejemplo, "Cómo tuniar una zanella. Hola gente hoy les voy a enseñar a tuniar una zanella”) -hechas por jóvenes con ciertas dificultades en el manejo de las normas ortográficas del castellano, como se puede observar en algunos de ellos.

\section{LAS TENDENCIAS PALATALIZADORAS}

DE LA LENGUA HABLADA: DE TUNIAR A TUÑAR

La diacronía de las lenguas romances explica que algunas vocales en hiato del latín, como consecuencia de la pérdida de la cantidad y la aparición del acento de intensidad, acaban convirtiéndose en semivocales muy relajadas, que llegan a desaparecer sin dejar rastro o permanecen para consonantizarse e influir en los sonidos vecinos. Según Väänänen (1971, p. 95), la semivocal era "una sonora constrictiva" cuya frecuencia en latín aumentó de forma notable por "la consonificación de $i$ y $e$ en hiato". De esta forma, las vocales palatales $e, i$, transformadas en la semiconsonante $/ \mathrm{j} /$, comienzan a "infectar a la consonante precedente”, según indica Alarcos (1971, p. 232). El paso siguiente consistirá en la supresión de este elemento que habría dejado huella "en forma de rasgo de palatalidad" en la consonante anterior, como ilustran los casos de SOMNIU > sueño, SENIORE $>$ señor. 
En ellos el segmento consonántico se ve modificado, puesto que, por la influencia del sonido siguiente, retrasa su punto de articulación y asimila el de la semiconsonante palatal que le sigue. Este proceso da origen al fonema palatal nasal sonoro /n/ del castellano y de otros romances, que se genera en abundantes vocablos por el contacto asimilador del fonema $/ \mathrm{n} /$ alveolar nasal sonoro con la yod, articulación palatal extremadamente cerrada, de carácter semivocálico o semiconsonántico, que influye de dos formas: palataliza las consonantes y cierra en un grado (o inflexiona) las vocales con las que va en contacto (Barra Jover 1994).

La secuencia gráfica " $\mathrm{n}+\mathrm{i}+$ vocal” se registra abundantemente en latín, en vocablos como seniore, compania, hispania, somniu, que llevan el grupo en sus bases etimológicas o en otros que lo desarrollan en el latín hablado por ruptura del hiato, con cierre del elemento palatal y evoluciones idénticas (aranea, castanea, vinea). Esto indica que el gran foco generador del fonema palatal nasal sonoro $/ \mathrm{n} / \mathrm{en}$ el paso del latín al romance fue el contacto entre el fonema /i/ y la vocal que le seguía, como muy bien explica Ramón Mariño Paz cuando aduce que en este contexto la resonancia nasal de la vocal palatal de mínima abertura, procedente de la asimilación con la consonante nasal anterior o posterior, creaba condiciones idóneas para que el hiato vocálico, como estructura silábica muy inestable por naturaleza, se rompiese mediante el desarrollo de una consonante nasal palatal reforzadora del ataque de la segunda sílaba. Este desarrollo consonántico queda confirmado por los más antiguos documentos en romance (Mariño Paz 2017, p. 352).

Por lo demás, la mayoría de los vocablos mencionados coincide en el resultado palatal nasal sonoro con las palatalizaciones del grupo GN, como consecuencia del proceso de debilitamiento y la posterior semiconsonantización del fonema velar oclusivo sonoro /g/ en situación implosiva o coda silábica: COGNATU > cuñado; LIGNA > leña; PUGNU > puño; TAM MAGNU $>$ tamañ $o^{12}$. Ambos contextos invitan a afirmar que este fonema palatal sonoro nasal se generó como consecuencia del contacto asimilador del fonema alveolar nasal sonoro con la yod posterior o anterior:

$$
\begin{aligned}
& \mathrm{NY}>\mathrm{nj}>\mathrm{n}: \text { SOMNIU }>\text { sueño ['sweno] } \\
& \mathrm{NE}+\mathrm{VOCAL}>\mathrm{nj}+\mathrm{vocal}>\mathrm{nj}>\mathrm{n}: \mathrm{CUNEA}>\mathrm{cuña} \text { ['kuna] } \\
& \mathrm{GN}>\mathrm{YN}>\mathrm{jn}>\mathrm{n}: \text { LIGNA }>\text { leña ['lena] }
\end{aligned}
$$

Sobre la cronología de estos cambios, en su Manual de gramática histórica española, Menéndez Pidal (1985 [1904], p. 47) habla de

12 También coincide, aunque por vía diferente, con el fonema palatal nasal sonoro que proviene de la degeminación de la alveolar nasal sonora /n/ ( $\mathrm{NN}>\mathrm{n}$; ANNUCUlu > anoxo) y, en ocasiones, de -mn- (Fradejas 1997, p. 103). 
la "yod segunda nasal" y explica que sus efectos palatalizadores suceden en fechas muy tempranas: se dan en el latín vulgar y podría afirmarse que tienen una dimensión panrománica, porque sus huellas se rastrean en todas las lenguas derivadas del latín (Ariza 2012, p. 28). Formas como CASTANEA > castaña, VINEA > viña, ARANEA > araña, EXTRANEU > extraño, STAMINEA > estameña, AD LATANEU > aledaño, y muchas otras, se originaron gracias a procesos de palatalización nasal motivados por rupturas de hiatos. Otras como sOMNIU > sueño, SENIORE $>$ señor O HISPANIA > España, que actúan de la misma forma, llevan el grupo NY en sus raíces latinas.

Si se traslada el proceso descrito al español hablado de hoy, se comprobará que aún es posible encontrar testimonios de evoluciones similares: el hipocorístico Toño a partir de Antonio y su variante femenina Toña, al lado de las formas plenas Antoño y Antoña, o el sustantivo coloña a partir del cultismo colonia son habituales en el habla viva. Algunas de estas formas tienen una fuerte presencia en el ámbito hispano, por ejemplo en Ecuador, donde Carlos Joaquín Córdova (1996, p. 195) atestigua abundantes ejemplos de "palatalización del grupo Ni seguido de vocal". El profesor ecuatoriano habla de "barbarismos fonéticos", con ejemplos como ñeto por nieto, ñeve por nieve, matrimoño por matrimonio y el ya citado Antoño por Antonio. Todos proceden de términos que han conservado el grupo NY sin palatalizar en el castellano general y sirven de paradigma a la variante tuñar, creada en algunas regiones del español por la palatalización del grupo NY surgido de la ruptura del hiato originario del verbo (tunear) gracias al cierre de su vocal palatal /e/, como se ha apuntado más arriba.

La que nos ocupa no es una solución habitual en el modelo estándar del castellano, sino un nuevo reflejo de la extraordinaria variación que afecta a nuestra lengua en sus diferentes latitudes ${ }^{13}$. En este caso, formas relacionadas con el paradigma de conjugación del verbo tuñar muestran las variantes intermedias tuñeary tuñiar, que se localizan preferentemente en el habla popular argentina, con presencia esporádica en otras regiones del idioma. Tuñar se usa con plena conciencia de su carácter palatal en la publicación de Facebook de una mujer bonaerense de mediana edad, quien realiza un montaje a partir de la etiqueta de una botella de coñac de la marca "Reyes Magos". En su creación artística, aparece modificada con el rostro de un individuo montado a caballo, como en la figura original de la etiqueta; el

13 Como afirma Assumpció Rost Bagudanch (2011, p. 373), "el habla formal está constreñida por las circunstancias y viene acorazada por la norma, huye de localismos y de realizaciones que se apartan de la prescripción; en consecuencia, no siempre se corresponde con la lengua viva y dinámica, la empleada en las relaciones diarias de la calle, que es la que, en definitiva, decide las evoluciones". Esta reflexión podría aplicarse al papel que representa en el español vivo de nuestros días la variante tuñar, con palatalización de su primitivo grupo $\mathrm{NY}+$ vocal. 
texto que la acompaña es la expresión "lo tuñé!!!"14, que corresponde a la primera persona del singular del pretérito perfecto simple de indicativo del verbo tuñar tras la palatalización -previa ruptura del hiato- de tunear. Ejemplos similares se encuentran dispersos por la red, casi siempre haciendo referencia a los cambios que se pueden introducir en vehículos de tracción mecánica o en videojuegos, todos ellos localizados en Argentina, sin excepciones:

1. Cómo tuñar un auto.

2. Roblox Tuñar Tu Coche Gratis! Hola Redzonitos: en Este video Enseñaré cómo Tuñar los autos gratis no importa el dinero que tengas.

3. Trabajo en tuñar autos.

4. Primero la tengo que tuñary aprenderme los truquitos.

5. Juegos play 2 tuñar.

6. Juegos de autos para tuñar.

7. Aprendí a tuñar autos con photoshop!!!!!!!!!!!

8. Bueno ésta es la primera foto que tuñeo así que espero que les guste de paso pueden jugar un rato encontrándole las diferencias jajaja bue me voy cuídence.

Estos ejemplos, y otros semejantes, muestran que la forma palatalizada del anglicismo tiene una fuerte presencia en el habla argentina actual. Entre ellos, tuñeo se muestra como una mezcla de la tendencia innovadora que representa la palatalización y la conservadora del hiato original. Los comentarios que se leen en algunos de los vídeos digitales a los que corresponden las descripciones anteriores pueden contener reflexiones metalingüísticas, como uno que sugiere que la variante palatalizada se estima como forma incorrecta; en otro foro, una mujer mexicana aclara que "es tunear no tuñar", ejemplo claro del carácter restrictivo de su empleo y de las connotaciones sociolingüísticas que se le asocian.

Al lado de tuñar se han localizado otras dos variantes que interesan como eslabones en el paso del anglicismo tunear a tuñar: tuñear y tuñiar. La primera, como se apuntó más arriba, muestra fundidos en su significante los fenómenos de la palatalización y la conservación (gráfica, al menos) del hiato; el segundo es una simbiosis del hiato destruido y de la palatalización del fonema alveolar nasal que

${ }^{14}$ Véase https://www.facebook.com/teresa.sarandon (consultado el 10 de enero y el 24 de mayo de 2018). 
le sigue. Del primero se han constatado 15 casos, en su mayoría presentes en vídeos grabados por jóvenes argentinos hábiles en las técnicas de modificación de vehículos y de escenas de videojuegos:

1. Trabajo en tuñear autos (Argentina).

2. Cómo tuñear autos en el nefor espid carbono para p. [Y las variantes tuñeamos, tuñeando, "déjame tuñearlo a mí”] (Argentina).

3. Luego de las vacaciones! Tuñear mis manos! Lo mejor de la vida! (Argentina).

4. Es un gran auto pero lo tienes que tuñear así aumenta su velocidad así llegar tranquilo al torneo (Argentina).

5. Que habían observado películas torrent cartelera nervión juegos de tuñear autos en algunos lugares del se intentó importar diversas veces (sin ubicación).

6. Y como acabé de tuñear, aquí lo dejé (Islas Canarias).

7. Perfecto... más que perfecto, no se explica el porqué se le tiene que tocar más y mucho menos tuñear (sin ubicación).

8. Gratis juegos de tuñear autos y otros que conseguir (sin ubicación).

9. Hobra de harte > dibujoh para tuñear el coshe (sin ubicación).

10. Re copada la camioneta yo la voy a tuñear así en gta 5 y el auto italiano (Argentina).

11. Es para tuñear el coche (Argentina).

12. Lokazo el de swat si se puede tuñear (Argentina).

La forma tuñiar proporciona también algunas referencias como las que se reproducen a continuación:

1. Cómo tuñiar collares de cuero para perros (niño argentino).

2. Cómo tuñiar el auto desde cualquier lado sin robux y sin garaje (Argentina).

3. Gastamos 5 millones en tuñiar un auto gta (niño argentino).

4. Los que se quieran tuñiary quedar pimp pimp (San Justo, Argentina). 
5. tuñiar fiat 600 (Argentina, con dos referencias).

6. tuñiar buby (Argentina).

7. ¿qué tan robado es un honda civic en méxico? Se puede tuñiar el Peugeot 404? (Argentina).

8. Disculpe mi pama usted vende las micas para tuñiar los controladores (Ecuador).

9. Cómo tuñiar autos (Argentina).

10. cómo tuñiar tu auto (Argentina).

11. Meter autos deportivos, que hubiese más forma de tuñiar el auto osea eligiendo alerones, capo, llantas, arreglar uno mismo el motor meterse hacer uno mismo (sin ubicación).

12. Cómo tuñiar el auto desde cualquier lado sin robux y sin garaje ( $\sin$ ubicación).

13. Cómo tuñiar collares de cuero (México).

De las trece referencias apuntadas, nueve proceden de Argentina, una de Ecuador y otra de México. Las dos restantes no ofrecen pistas claras para ubicarlas geográficamente, como refleja la siguiente tabla:

TABLA 2

La variante tuñiar

\begin{tabular}{lc}
\hline Argentina & $9(69.23 \%)$ \\
Ecuador & $1(7.69 \%)$ \\
México & $1(7.69 \%)$ \\
Sin ubicación & $2(15.38 \%)$ \\
Total & 13 \\
\hline
\end{tabular}

Las formas anteriores permitirían trazar un proceso evolutivo que recuerda al operado en el paso del latín vulgar al castellano: TUNEAR $>$ [tu'njar $]>$ [tu'njar $]>$ [tu'nar $]$. Cabe afirmar que en el español hablado actual, el préstamo adaptado del inglés en la forma tunear se ve sometido a la presión de la lengua hablada y modifica la articulación de su hiato. En medio de la tensión entre la oralidad y la escritura, la semiconsonante palatal que resulta de la diptongación del hiato transfiere sus rasgos articulatorios al fonema alveolar nasal /n/ y lo palataliza; en esta innovación, la yod resultante de la tendencia antihiática traslada sus rasgos articulatorios al fonema 
consonántico y se mantiene sin fundirse con él. En el último estadio, la asimilación de los dos sonidos palatales vecinos lleva hasta la variante final tuñar. Queda en el camino una forma híbrida entre las tendencias conservadoras de hiato y la palatalización del fonema consonántico nasal, tuñear, habitual en registros orales y escritos del español de Argentina, con un caso documentado también en las Islas Canarias. Según muestra en su significante la grafía que representa en castellano el fonema palatal nasal sonoro $/ \mathrm{n} /$, habría que interpretarla como el resultado de una combinación (tal vez ultracorrecta) entre el étimo (el préstamo adaptado tunear) y el resultado con palatalización, previa ruptura del hiato.

\section{REFLEXIONES FINALES}

Los fenómenos analizados ponen en evidencia la enorme presión que ejercen los códigos de la oralidad en el sistema lingüístico del español actual. En esta época de flujo intenso de anglicismos, muchos de los cuales se asientan en la lengua con mínimas variaciones en su forma, llama la atención que un vocablo como tunear se haya visto sometido en determinadas áreas del ámbito hispano a transformaciones profundas en su forma y en su articulación. Que un anglicismo se adapte a las normas del español actual no es sorprendente: es, más bien, un dictado de la norma estándar de la lengua que busca acomodar los préstamos que recibe a estructuras conocidas por el sistema que los acoge. Y eso es lo que se ha hecho con tunear a partir de su étimo inglés to tune. Bajo esta apariencia, ha coincidido con un vocablo de significado relativamente arcaizante desde la perspectiva del presente ('vivir como un tuno o pícaro') o de uso restringido (el mexicanismo tunear, 'cosechar higos de tuna', adscrito en exclusiva al ámbito de uso del país americano). Es decir, ha ocupado un lugar en el inventario del léxico del español con una forma asimilada por la tradición pero desprovista en el presente de su significado primitivo.

En esta andadura, desde su aparición hasta llegar a la variante palatalizada, ha recorrido un largo camino en que el principal eslabón ha sido la ruptura del hiato mediante el cierre de la vocal $e$. De ahí a la palatalización ha dado un paso similar al que dieron muchas palabras en la evolución del latín al romance. Y, como ellas, ha resuelto el conflicto de las dos vocales unidas en sílabas separadas mediante la conversión de una de ellas en semiconsonante palatal. Como también pasó en el latín hablado, esa yod semiconsonántica ha retrasado el punto de articulación del fonema alveolar nasal sonoro hacia la zona del paladar y se ha fundido con ella sin dejar rastro del primitivo grupo vocálico que constituía dos de sus sílabas. No podemos averiguar qué pasará en el futuro, si triunfarán las formas tuniar, tuñiar, 
tuñear y tuñar, hoy estigmatizadas por la presión del uso culto -pensemos que algunas de las formas ofrecidas por el CORPES XXI contienen reflexiones metalingüísticas que hacen referencia al registro o a las cotas que alcanza el uso de este vocablo: "Coloquialmente, ¿tuneamos a Marsé?”, en un folleto divulgativo publicado por la Universidad de Alcalá de Henares para presentar el Festival de la palabra en 2009, o "Lo que ahora llamamos despectivamente tuneo" (España, 2015), en un reportaje de divulgación.

Lo que sí está claro es que este préstamo adaptado del inglés muestra un polimorfismo intenso en el habla y está sujeto a procesos de variación que, cuando se disponga de un mayor número de ocurrencias, proporcionará más datos sobre sus ámbitos de uso y sobre las condiciones socioculturales de sus usuarios. De momento, permite constatar el hecho de que la lengua hablada sigue mostrando fenómenos similares a los que se dieron en el paso del latín a las lenguas románicas, en esa versión coloquial, hablada, de la lengua culta de Roma que tantas pistas ha proporcionado a los historiadores de la lengua para comprender los procesos de cambio operados en la evolución de los diferentes romances. Habrá que discutir el verdadero alcance del fenómeno, teniendo en cuenta que los ejemplos que se han allegado para explicar el proceso de palatalización en la lengua latina permiten interpretar un cambio fonológico culminado, basado en documentos escritos de diverso signo y analizados por los estudiosos de esa modalidad hablada del latín, en tanto que la propuesta que se hace para explicar la evolución tunear $>$ [tu'njar] $>$ [tu'njar] $>$ [tu'nar] se basa en una muestra de formas dispersas halladas, fundamentalmente, en publicaciones digitales de diferentes países cuyo ingreso en el sistema aún está por ver.

\section{REFERENGIAS}

\section{Corpus}

CORDE $=$ Real Academia Española: Banco de datos (CORDE) [en línea]. Corpus diacrónico del español, http://www.rae.es [consultado en marzo de 2018].

CORPES XXI = Real Academia Española: Banco de datos (CORPES XXI) [en línea] . Corpus del español del Siglo XXI (CORPES), http:/ / www.rae.es [consultado en marzo de 2018].

CREA = Real Academia Española: Banco de datos (CREA) [en línea]. Corpus de referencia del español actual, http://www.rae.es [consultado en marzo de 2018].

\section{Repertorios lexicográficos}

Asociación de Academias de la Lengua Española 2010. Diccionario de americanismos, Santillana, Madrid, http.//www.rae.es [consultado el 14 de mayo de 2018]. 
$D L E=$ Real Academia Española y Asociación de Academias de la Lengua Española 2014. Diccionario de la lengua española, $23^{\mathrm{a}}$ ed., http.//www.rae.es [consultado en marzo de 2018].

Real Academia Española. Nuevo tesoro lexicográfico del español, http://buscon.rae.es/ ntlle/SrvltGUILoginNtlle [consultado en marzo de 2018].

Real Academia Española y Asociación de Academias de la Lengua Española 2005. Diccionario panhispánico de dudas, Santillana, Madrid, http.//www.rae.es [consultado el 14 de mayo de 2018].

Seco, Manuel, Olimpia Andrés y Gabino Ramos 2018 [1999]. Diccionario de uso del español actual, Aguilar, Madrid.

\section{Referencias bibliográficas}

Aguilar, Lourdes 1999. "Hiatus and diphthong: Acoustic cues and speech situation differences", Speech Communication, 28, pp. 57-74.

Aguilar, Lourdes 2006. "A propósito de las combinaciones vocálicas", Nueva Revista de Filología Hispánica, 54, pp. 353-381.

Alarcos Llorach, Emilio 1971. Fonología española, Gredos, Madrid.

Alcoholado, Antonio 2012. "La tendencia antihiática del español: descripción, uso en registros formales y proyección en la enseñanza de ELE”, RedELE, 24, hdl: http://hdl.handle.net/11162/94408.

Alcoholado, Antonio 2013. "Vocales en contacto en la expresión oral del español. Planteamientos teóricos y perspectivas de trabajo con estudiantes universitarios chinos de español como lengua extranjera", SinoELE (Suplementos, 8), http:/ / www.sinoele.org/index.php/revista/suplementos?id=202 (consultado el $11 \mathrm{de}$ enero de 2018 y el 27 de marzo de 2019).

Alvar, Manuel 1996. "Paraguay", en Manual de dialectología hispánica. El español de América. Coord. Manuel Alvar, Ariel, Barcelona, pp. 196-208.

Alvar, Manuel (coord.) 1996a. Manual de dialectología hispánica. El español de América, Ariel, Barcelona.

Ariza Viguera, Manuel 2012. Fonología y fonética históricas del español, Arco/Libros, Madrid.

Barra Jover, Mario 1994. "Reflexiones sobre el concepto de yod", Revista de Filología Española, 74, 3/4, pp. 181-188.

Colantoni, Laura \& Anna Limanni 2008. "Where are hiatuses left? A comparative study of vocalic sequences in Argentine Spanish”, en Romance Linguistics 2008. Interactions in Romance. Eds. Karlos Arregi, Zsuzsanna Fagyal, Silvina A. Montrul \& Annie Tremblay, John Benjamins, Amsterdam-Philadelphia.

Córdova, Carlos JoaQuín 1996. "Ecuador", en Manual de Dialectología Hispánica. El español de América. Coord. Manuel Alvar, Ariel, Barcelona, pp. 184-195.

Fradejas Rueda, José Manuel 1997. Fonología histórica del español, Visor, Madrid.

Lipski, John M. 1996. El español de América, Cátedra, Madrid.

Lope Blanch, Juan M. 1996. "México", en Manual de dialectología hispánica. El español de América. Coord. Manuel Alvar, Ariel, Barcelona, pp. 81-89.

Mariño Paz, Ramón 2017. Fonética e fonoloxía históricas da língua galega, Edicións Gerais de Galicia, Vigo.

Martín García, Josefa 2007. "Verbos denominales en -ear. caracterización léxico-sintáctica”, Revista Española de Lingüistica, 37, pp. 247-278, doi: 10.31810/RSEL.37.1.

Menéndez Pidal, Ramón 1985 [1904]. Manual de gramática histórica española, Espasa Calpe, Madrid.

Montero Curiel, Pilar en prensa. "Reflexiones sobre la diptongación del hiato en español", en Aportaciones y retos de la tradición cultural hispánica en una sociedad 
global. Coords. Kyra A. Kietrys, María Luisa Montero, Carmen Sotomayor y Adam L. Winkel, Servicio de Publicaciones de la Universidad de Extremadura, Cáceres. Montes Giraldo, José Joaquín 1996. "Colombia", en Manual de dialectología hispánica. El español de América. Coord. Manuel Alvar, Ariel, Barcelona, pp. 134-145.

Navarro Tomás, Tomás 1918 [2004]. Manual de pronunciación española, CSIC, Madrid. $N G L E=$ Real Academia Española y Asociación de Academias de la Lengua Española 2009. Nueva gramática de la lengua española. Morfología y sintaxis, Espasa Calpe, Madrid.

Pena, Jesús 1980. La derivación en español. Verbos derivados y sustantivos verbales, Universidade de Santiago de Compostela, Santiago de Compostela. (Anejos de Ver$b a, 16)$.

Pena, Jesús 1993. "La formación de verbos en español: la sufijación verbal", en $L a$ formación de palabras. Ed. Soledad Varela, Taurus, Madrid, pp. 217-281.

Quesada Pacheco, Miguel Ángel 1996. "El español de América Central”, en Manual de dialectología hispánica. El español de América. Coord. Manuel Alvar, Ariel, Barcelona, pp. 101-115.

Quilis Morales, Antonio 1999. Tratado de fonología y fonética españolas, Gredos, Madrid.

Quilis Morales, Antonio 2005. Fonética histórica y fonología diacrónica, UNED, Madrid.

Rost Bagudanch, Assumpció 2011. Variación en los procesos de palatalización de yod segunda (o cómo la sincronía permite la explicación de la diacronía), tesis, Universitat de Girona, https://www.tdx.cat/handle/10803/31860 (consultada en enero y mayo de 2018).

Sedano, Mercedes y Paola Bentivoglio 1996. "Venezuela", en Manual de dialectología hispánica. El español de América. Coord. Manuel Alvar, Ariel, Barcelona, pp. 116-133.

Serrano Dolader, David 1999. "La derivación verbal y la parasíntesis", en Gramática descriptiva de la lengua española. Eds. Ignacio Bosque y Violeta Demonte, Espasa Calpe, Madrid, t. 3, pp. 4683-4755.

VÄÄNÄNEN, VEIKKo 1971. Introducción al latín vulgar, Gredos, Madrid.

Vaquero, María 1996. El español de América. T. 1: Pronunciación. Arco/Libros, Madrid. VAQUero, MARía 1996a. "Antillas", en Manual de dialectología hispánica. El español de América. Coord. Manuel Alvar, Ariel, Barcelona, pp. 51-67.

\section{APÉNDICE}

Procedencia de los ejemplos:

De tuniar:

1. https://www.taringa.net/posts/autos-motos/5221462/Como-tuniar-una-zanella50cc.html (consultado el 14 de enero de 2018).

2. http://www.de-venta.com.ar/aviso-652052-vendo-fiat-para-coleccionista-o-paraaquellos-que-le-gusta-tuniar-veiculos-villa-union (consultado el 14 de marzo de 2019).

3. https://cordobacapital.oix.com.ar (consultado el 14 de mayo de 2018).

4. https://cenationgames7347.webnode.cl/como-tuniar-tu-auto-de-gta-sanandreas/ (consultado el 14 de marzo de 2019).

5. https://www.articulo.mercadolibre.como.co (consultado el 14 de mayo de 2018). 
6. https://inmuebles.mercadolibre.com.uy/se-vende-auto-ideal-para-tuniar (consultado el 14 de enero de 2018).

7. https://computacion.mercadolibre.com.uy/barras-para-tuniar-tu-auto (consultado el 14 de enero de 2018).

8. https:/ / www.youtube.com/watch?v=hEkL_Fa038w (consultado el 14 de mayo de 2018).

9. https://youtube.com/watch?v=_iCKqVy7G1c (consultado el 13 de marzo de 2019).

10. https://www.youtube.com/watch?v=scNDAL15mE4 (consultado el 13 de marzo de 2019).

11. https://www.youtube.com/watch?v=Xgt8LLxVk18, 31 de diciembre de 2017 (consultado el 13 de marzo de 2019).

12. https://valvulita.com (consultado el 14 de mayo de 2018).

13. https://razine.com (consultado el 14 de mayo de 2018).

14. (25 de enero de 2015) y "Como tuniar autos en gta5", ambos ejemplos en una página de "customización" ubicada en la ciudad de Los Santos, en California (Los Santos Customs) (17 de febrero de 2017). (Consultado el 13 de marzo de 2019).

15. Arturo Pérez Reverte, "La venganza de Churruca", Cuando éramos honrados mercenarios (2005-2009), http://www.perezreverte.com/upload/ficheros/ libros/201001/cuando_eramos_honrados_mercenarios.pdf (consultado el 14 de enero de 2018).

16. https://www.youtube.com/watch?v=XBN4ce7oWAI (consultado el 14 de marzo de 2019).

17. https://www.youtube.com/watch?v=v9TSGF00SPc 2017 (consultado el 13 de marzo de 2019).

De tuñar:

1. https://www.youtube.com/watch?v=ErWL0VyaltI (consultado el 10 de enero de 2018).

2. https://www.youtube.com/watch?v=MgQiWjSh9D8 (consultado el 10 de enero de 2018).

3. https://www.facebook.com/pages/trabajo-en-tuñar-autos/244636325595861 (consultado el 10 de enero de 2018).

4. https://www.taringa.net/comunidades/bikeringa/1801896/GENTEEEEEEEEEEE.html (consultado el 10 de enero de 2018).

5. https://listado.mercadolibre.com.ar/juegos-play-2-tuñar (consultado el 10 de enero de 2018).

6. https://www.juegosipo.com/juegos-de-autos-para-tuñar/ (consultado el 10 de enero de 2018).

7. http://www.fotolog.com/lacrimosa_12/ (consultado el 10 de enero de 2018).

8. http://www.fotolog.com/lacrimosa_12/ (consultado el 10 de enero de 2018).

De tuñear:

1. https:/ / www.facebook.com/pages / trabajo-en-tu \% C3\%B 1 earautos/142038769236567 (consultado el 14 de marzo de 2019); https://www. youtube.com/watch?v=rTyWBVjb-7g (consultado el 13 de marzo de 2019).

2. http://picdeer.com/normazvera (consultado el 13 de marzo de 2019).

3. https://www.3djuegos.com/foros/tema/1464978/0/ayuda-para-elegir-coche/ (consultado el 13 de marzo de 2019).

4. https://www.3djuegos.com/foros/tema/1464978/0/ayuda-para-elegir-coche/ (consultado el 14 de marzo de 2019).

5. https://implantadococlear.blogspot.com/2011/05/dia-855-un-par-de-tres-noticias.html (consultado el 14 de marzo de 2019). 
6. http://foro.clubjapo.com/t/nuevo-celica-biplaza-con-motor-central/14878/30 (consultado el 14 de marzo de 2019).

7. http://dvixtotal9.10bet.ovh/dvixtotal-bajar-musica-gratis/?c=juegos-detu\%C3\%B1ear-autos_f=m_19005-2 (consultado el 14 de marzo de 2019).

8. https://pon.mforos.com/1551165/9570511-evangelion-o-naruto/?pag=3 (consultado el 14 de marzo de 2019).

9. https://ch-me.org/videos/wallride-super-imposible-carrera-gta-v-online-gta-5-online-LOLQjmMsvW8.html (consultado el 14 de marzo de 2019).

10. https://ch-me.org/sname/polisycacos (consultado el 14 de marzo de 2019).

11. https://www.clipsoon.com/I0U7AEPUiYs/mrlokazo86.html (consultado el 14 de marzo de 2019).

De tuñiar:

1. https://www.youtube.com/watch?v=rnB9VceVW9A (consultado el 14 de marzo de 2019).

2. https://www.youtube.com/watch?v=KGAf2oeUKtY (consultado el 14 de marzo de 2019)

3. https://www.youtube.com/watch?v=9fjd9TBjyXM (consultado el 14 de marzo de 2019).

4. https://www.facebook.com/doctorloveeeeeee/posts/aca-en-palomarlos-que-se-quieran-tu \% C3\% B 1 iar-y-quedar-pimp-pimp-nos-vinimos-conhome/830218877165064/ (consultado el 14 de marzo de 2019).

5. https://deportes.mercadolibre.com.ar/tu\%C3\%B1iar-fiat-600 (consultado el 14 de marzo de 2019).

6. https://coleccionables.mercadolibre.com.ar/vehiculos-miniatura/autos/buby/ tu\%C3\%B1iar-fiat-600 (consultado el 14 de marzo de 2019).

7. https://0es.org/403.shtml (consultado el 14 de marzo de 2019).

8. https://www.ocompra.com/ecuador/item/audifono-teclado-mouse-gamer-havitmousepad-original-luz-led-413010317/ (consultado el 14 de marzo de 2019).

9. https://by-clips.com/channel/UCyCDSVw-ToaqL1AWCfPGuNw (consultado el 14 de marzo de 2019).

10. https://by-clips.com/channel/UC08ESCEkRcp4H5kcMLbbpHQ (consultado el 14 de marzo de 2019).

11. http://androideverde.pro/es/g/9995/Drift+Horizon+Online (consultado el 14 de marzo de 2019).

12. http://newsmdn.com/tag/cualquier-lado/CJMBEAA (consultado el 14 de marzo de 2019).

13. https://mx.ytb.lv/search/collar+adiestramiento.html (consultado el 14 de marzo de 2019). 